\title{
The simulation of gas-dynamic characteristics of centrifugal compressors in turbo-expander units
}

\author{
$A$. Rekstin ${ }^{1, *}, V$. Semenovskiy ${ }^{1}, K$. Soldatova ${ }^{1}, Y$. Galerkin ${ }^{1}$, and $K$. Sokolov ${ }^{2}$ \\ ${ }^{1}$ Peter the Great St. Petersburg Polytechnic University, St. Petersburg, Russia \\ ${ }^{2}$ The JSC "Turbokholod", Moscow, Russia
}

\begin{abstract}
Prof. Y. Galerkin and his team have developed and completed designs of 19 single-stage centrifugal compressors for turbo-expander packaged units applying Universal Modeling Method for the company "Turbokholod JSC" since 2005. The most powerful compressor is $6500 \mathrm{~kW}$. The highest delivery pressure is $12 \mathrm{MPa}$. One hundred fifteen turbo-expander packaged units with total capacity $400000 \mathrm{~kW}$ were manufactured, installed and were in operation in December 2018. The gas-dynamic characteristics of compressors comply with technical specification when operated within given range of initial temperatures and initial and final pressures - up to 16 combinations for some compressors. The dimensionless characteristics of the compressor stages vary within the range of design parameters: flow rate coefficient $0.0278-0.0697$, loading factor $0.43-0.71$. The simulation of gas-dynamic characteristics of one of the designed compressors by the newest version of mathematical model is presented as an example, demonstrating the simulation features and effectiveness.
\end{abstract}

\section{Introduction}

Gas cooling turboexpander units operate at pipelines year-round in the permafrost areas [1]. Turboexpanders [2] are also widely used as sources of refrigeration in industrial processes such as the extraction of ethane and natural-gas liquids (NGLs) from natural gas. Some important features of the compressors are presented below:

- the compressors are centrifugal and single-stage,

- vane diffusers are applied as a constructive element, not always necessary from the aerodynamic point of view,

- the impellers are of traditional 2-D type,

- pressure ratio lies in range $1.07-1.50$ in most cases. Circumferential velocity reaches $340 \mathrm{~m} / \mathrm{s}$,

- impellers are cast. The considerable roughness of the surface and some shape irregularity take place.

The team of the R\&D Laboratory "Gas dynamics of turbo machines" has a lengthy experience of centrifugal process compressor design $[3,4,5,6,7]$. Among the designs produced, there are fifteen types of turboexpander units with compressors designed by Universal modeling method. The specific features of these compressors were not reflected by early versions of the math models. The designers' experience and intuition led to successful designs in the past. The aim of the work is to prove the new 8 th version $[8,9]$ of the math model ability to reflect specific features of the turboexpander unit compressor without individual tuning of the model.

\section{Design experience of centrifugal compressors for turboexpander units}

Table 1 provides information on compressors after designs of prof. Yu. Galerkin and his team.

One of the design principles of the Universal Modeling Method $[4,5,10]$ is that calculations are dimensionless. The main design parameters of the stage, determining the effectiveness and characteristics configuration are:

- design flow rate coefficient:

$$
\Phi_{\text {des }}=\frac{\bar{m}}{\rho_{0}^{*} 0.785 D_{2}^{2} u_{2}}
$$

- design loading factor:

$$
\psi_{T \text { des }}=c_{\mathrm{u} 2} / u_{2}
$$

The combination of these coefficients predetermines the value of the specific speed. The specific speed points on the possibility or impossibility of designing a compressor with favorable gas-dynamic characteristics. The specific speed is [4]:

$$
K_{n}=0.0591\left(\bar{m} / \rho_{0}^{*}\right)^{0,5} / h_{T}^{0.75}=\Phi^{0.5} / \psi_{T}^{0.75}
$$

Theoretical considerations and design practice demonstrate the following [5]:

\footnotetext{
*Corresponding author: swb@neva.ru
} 
Table 1. Summary table of the designed compressors.

\begin{tabular}{|l|c|c|c|c|c|c|c|c|}
\hline & $\begin{array}{c}\text { Flow } \\
\text { rate }\end{array}$ & $\begin{array}{c}\text { Delivery } \\
\text { pressure }\end{array}$ & $\begin{array}{c}\text { Pressure } \\
\text { ratio }\end{array}$ & RPM & $\begin{array}{c}\text { Impeller } \\
\text { diameter }\end{array}$ & $\begin{array}{c}\text { Flow rate } \\
\text { coefficient }\end{array}$ & $\begin{array}{c}\text { Loading } \\
\text { factor }\end{array}$ & $\begin{array}{c}\text { Specific } \\
\text { speed }\end{array}$ \\
\hline & $(\bar{m}, \mathrm{~kg} / \mathrm{s})$ & $(\mathrm{MPa})$ & $\left(\pi^{*}\right)$ & $\left(n, \mathrm{~min}^{-1}\right)$ & $D_{2}, \mathrm{~m}$ & $\Phi_{\text {des }}$ & $\psi_{\text {Tdes }}$ & $K_{n}$ \\
\hline TK1 & 59.0 & 11.5 & 1.278 & 15500 & 0.320 & 0.0339 & 0.614 & 0.265 \\
\hline TK2 & 79.0 & 7.8 & 1.300 & 15500 & 0.365 & 0.0339 & 0.542 & 0.291 \\
\hline TK3 & 78.1 & 8.906 & 1.220 & 14000 & 0.365 & 0.0508 & 0.430 & 0.424 \\
\hline TK4-0.53 & 79.0 & 6.75 & 1.424 & 16250 & 0.39 & 0.0661 & 0.558 & 0.398 \\
\hline TK5 & 71.4 & 7.75 & 1.277 & 16000 & 0.35 & 0.0609 & 0.500 & 0.415 \\
\hline TK6 & 163.0 & 8.69 & 1.070 & 5300 & 0.575 & 0.0684 & 0.490 & 0.447 \\
\hline TK7 & 86.3 & 13 & 1.209 & 16000 & 0.275 & 0.0637 & 0.510 & 0.418 \\
\hline TK8 & 59.19 & 13 & 1.209 & 16000 & 0.275 & 0.0438 & 0.510 & 0.347 \\
\hline TK4-.70 & 79.0 & 6.75 & 1.424 & 14500 & 0.410 & 0.0576 & 0.710 & 0.310 \\
\hline TK10 & 51.2 & 13.2 & 1.320 & 16500 & 0.310 & 0.0278 & 0.613 & 0.241 \\
\hline TK11 & 103.5 & 12.2 & 1.208 & 15000 & 0.310 & 0.0632 & 0.510 & 0.417 \\
\hline TK12 & 60.5 & 13 & 1.244 & 15500 & 0.275 & 0.0450 & 0.573 & 0.322 \\
\hline TK13 & 85.19 & 12.53 & 1.171 & 13300 & 0.300 & 0.0600 & 0.550 & 0.383 \\
\hline TK14A & 84.0 & 12.5 & 1.202 & 12500 & 0.340 & 0.0417 & 0.527 & 0.330 \\
\hline TK15 & 88.91 & 5.513 & 1.0724 & 5100 & 0.490 & 0.0690 & 0.480 & 0.455 \\
\hline
\end{tabular}

- in the range of $\Phi_{\mathrm{des}}=0.055-0.075$, the maximum efficiency can be achieved. Out of the range the efficiency reduction is inevitable,

- low loading factor $\psi_{\text {Tdes }}=0.42-0.52$ leads to highest efficiency and allows for favorable characteristics.

The Universal modeling method, which is currently implemented as a PC software, operates as follows:

- the primary design is performed for a preselected efficiency taking into account similarity criteria and construction limitations, applying the rules and calculation formulae for the necessary $\Phi_{\text {des }}$ and $\psi_{\text {Tdes }}$, meaning that the form and the ratio of the dimensions of the flow path are determined;

- the efficiency of the primary design of the flow path is determined according to the mathematical model of the losses' calculation, and its dimensions are corrected if the preselected and calculated efficiencies do not match;

- some alternative candidates are created through variations of the dimension ratio of the flow path, the efficiency of which is calculated further. The corresponding optimization algorithm searches for the maximum efficiency version and relative flow path dimensions;

- after the optimization of the vane apparatus of the compressor based on the speed diagram of an inviscid flow, the mathematical model calculates the gasdynamic characteristics of the compressor.

-the necessary number of compressor candidates is calculated using the simplified Math model [11] that estimates any stage efficiency operating with $\Phi_{\text {des }}$ and $\psi_{\text {Tdes }}$ only,

- the primary design of the chosen candidate is performed by the set of specifically developed formulae [11],

- the math model of the primarily designed compressor characteristics are calculated by the Universal modeling. The mismatch with design parameters is eliminated and better efficiency is reached by flow part dimensions' variation,

- the family of exploitation characteristics is calculated by the Universal modeling math model.

Most of the compressor designs presented in the Table 1 are based on the 4th version of the mathematical model developed in the early 1990s [3]. A somewhat simplified physical model did not allow to get a set of empirical coefficients for an accurate modeling of stages with different design parameters $\Phi_{\text {des }}$ and $\psi_{\text {Tdes. }}$. It was necessary to create a different set of empirical coefficients for a specific combination of design parameters. Anyway, good designs were created thanks to the experience and intuition of the authors of the projects. The 15 compressors shown in the Table 1 operate at 12 gas fields. Their capacity lies in range of 7 to 20 million $\mathrm{m}^{3} /$ day. The most powerful compressor is $6500 \mathrm{~kW}$. The highest delivery pressure is $12 \mathrm{MPa}$. Their characteristics give the possibility to operate at different inlet conditions (up to 16 combinations given by the technical specification). The dimensionless characteristics of the stages cover the range design flow rate coefficient $0.0278-0.0697$ and the loading factor 0.43-0.71.

The newest designs are executed by the more sophisticated versions of the Math models. K. Soldatova [Ошибка! Источник ссылки не найден., 12Ошибка! Источник ссылки не найден.] has managed to develop a more perfect physical and mathematical model (version 5) and solve the problem of simulation of design regimes with only one universal set of empirical coefficients. Subsequent works [13, 14, 15] solved the problem of simulation of whole characteristics by a universal set of empirical coefficients. By the 4th -8 th Math model versions, several dozens of compressors have been designed for pipeline and other industries. About 400 compressors are constructed with total power of about 5 million $\mathrm{kW}$. These were big compressors with accurately mechanically treated flow parts - impellers are included. 
The turbo expander is comparatively small and their cast impellers have rough surface and certain irregularities of the flow path. It requires verification of the Math model.

\section{Test results}

The Turbokholod JSC Company executes compressor tests at the open loop test rig - air test. The other source of the information are measurements at compressor site gas test. To reduce test information, the following procedure is applied.

Constant values for a particular test:

Impeller diameter $D_{2} \quad(\mathrm{~m})$, average inlet-exit isentropic coefficient $k$, average inlet-exit gas constant $z R(\mathrm{~J} / \mathrm{kg} / \mathrm{K})$.

Measured data: rotor rpm $n$, inlet pressure $\left.p^{*}{ }_{\text {inl }}(\mathrm{Pa}),\right)$, exit pressure $p_{\text {ex }}^{*}(\mathrm{~Pa})$, inlet temperature $T^{*}{ }_{\text {inl }}\left({ }^{\circ} \mathrm{K}\right)$, exit temperature $T_{\text {ex }}^{*}\left({ }^{\circ} \mathrm{K}\right)$, mass flow $\bar{m}(\mathrm{~kg} / \mathrm{s})$.

Calculated data:

- circumferential velocity $(\mathrm{m} / \mathrm{s})$ :

$$
u_{2}=3.141 \cdot D_{2} \cdot n / 60
$$

- inlet gas density $\left(\mathrm{kg} / \mathrm{m}^{3}\right)$ :

$$
\rho_{\mathrm{inl}}^{*}=\frac{p_{\mathrm{inl}}^{*}}{(z R)_{\mathrm{inl}} \cdot T_{\mathrm{inl}}^{*}}
$$

- flow rate coefficient:

$$
\Phi=\frac{4 \bar{m}}{\rho_{\mathrm{inl}}^{*} \cdot 3.141 \cdot D_{2}^{2} \cdot u_{2}}
$$

- total pressure ratio:

$$
\pi^{*}=p_{\mathrm{ex}}^{*} / p_{\mathrm{inl}}^{*}
$$

- work coefficient:

$$
\psi_{\mathrm{i}}=\frac{c_{\mathrm{p}}\left(T_{\mathrm{ex}}^{*}-T_{\mathrm{inl}}^{*}\right)}{u_{2}^{2}}
$$

- total polytrophic efficiency:

$$
\eta^{*}=\left(\lg \pi^{*}\right)\left(\frac{k}{k-1} \lg \frac{T_{\mathrm{ex}}^{*}}{T_{\mathrm{inl}}^{*}}\right)^{-1}
$$

- polytrophic head coefficient:

$$
\psi_{\mathrm{p}}^{*}=\psi_{\mathrm{i}} \cdot \eta^{*}
$$

- impeller Mach number:

$$
M_{\mathrm{u}}=u_{2} / \sqrt{k \cdot z R \cdot T_{\mathrm{inl}}^{*}}
$$

Table 2 shows the results of measuring the parameters and calculating the efficiency and the pressure ratio of the compressor TK-4-530.
Table 2. Measured parameters of the compressor TK-4-530 on site.

\begin{tabular}{|c|c|c|c|c|}
\hline$\#$ & $\Phi$ & $\psi_{\mathrm{i}}$ & $\eta^{*} / \eta_{\max }^{*}$ & $\psi_{\mathrm{p}}^{*} / \psi_{\mathrm{p} \mathrm{opt}}^{*}$ \\
\hline 1 & 0.064 & 0.529 & 0.996 & 0.972 \\
\hline 2 & 0.066 & 0.540 & 0.999 & 0.994 \\
\hline 3 & 0.066 & 0.525 & 0.921 & 0.892 \\
\hline 4 & 0.067 & 0.539 & 0.755 & 0.750 \\
\hline 5 & 0.067 & 0.543 & 1.000 & 1.000 \\
\hline 6 & 0.068 & 0.517 & 0.921 & 0.877 \\
\hline 7 & 0.068 & 0.563 & 0.920 & 0.995 \\
\hline 8 & 0.069 & 0.538 & 0.999 & 0.991 \\
\hline
\end{tabular}

The measured data of the work coefficient and the efficiency gives an idea of the order of the quantities, but their scatter does not make it possible to use data to verify the mathematical model. Table 3 shows the measured and calculated parameters of the TK-4-530 compressor at the factory air tests.

Table 3. Measured parameters of the compressor TK-4-530 at the factory air test.

\begin{tabular}{|c|c|c|c|c|c|}
\hline № & $\mathrm{u}_{2, \mathrm{~m} / \mathrm{s}}$ & $\Phi$ & $\psi_{\mathrm{i}}$ & $\eta^{*} / \eta_{\max }^{*}$ & $\psi_{\mathrm{p}}^{*} / \psi_{\mathrm{p} \mathrm{opt}}^{*}$ \\
\hline 1 & 230.0 & 0.045 & 0.640 & 0.936 & 1.113 \\
\hline 2 & 230.0 & 0.045 & 0.640 & 0.944 & 1.122 \\
\hline 3 & 246.3 & 0.046 & 0.638 & 0.947 & 1.121 \\
\hline 4 & 246.3 & 0.046 & 0.639 & 0.945 & 1.122 \\
\hline 5 & 278.3 & 0.048 & 0.640 & 0.937 & 1.113 \\
\hline 6 & 278.3 & 0.048 & 0.640 & 0.938 & 1.114 \\
\hline 7 & 229.4 & 0.057 & 0.538 & 1.000 & 1.000 \\
\hline 8 & 229.4 & 0.057 & 0.538 & 0.995 & 0.995 \\
\hline 9 & 243.9 & 0.058 & 0.544 & 0.976 & 0.986 \\
\hline 10 & 243.9 & 0.058 & 0.546 & 0.974 & 0.987 \\
\hline 11 & 263.2 & 0.060 & 0.551 & 0.960 & 0.983 \\
\hline 12 & 263.2 & 0.060 & 0.551 & 0.961 & 0.984 \\
\hline 13 & 303.0 & 0.063 & 0.543 & 0.936 & 0.944 \\
\hline 14 & 303.0 & 0.063 & 0.543 & 0.937 & 0.944 \\
\hline
\end{tabular}

There are 7 points with different flow rate coefficients that are presented in the Table 2. These 2 data with good matching are presented at each $\Phi$. The functions $\eta^{*}, \psi_{\mathrm{i}}, \psi_{\mathrm{p}}^{*}=f(\Phi)$ are not performance characteristics of the compressor as the measurements were made at different impeller velocity $\left(M_{u}=0.67-\right.$ 0.87). But the configuration of the functions $\eta^{*}, \psi_{\mathrm{i}}$, $\psi_{\mathrm{p}}^{*}=f(\Phi)$ corresponds to compressor characteristics the maximum efficiency lies inside of the $\Phi$ range and work coefficient $\psi_{\mathrm{i}}=f(\Phi)$ is almost linear. The authors reckon that test data are reliable and can be used for the new $8^{\text {th }}$ version $[16,17]$ of the Universal modeling method math model verification. 


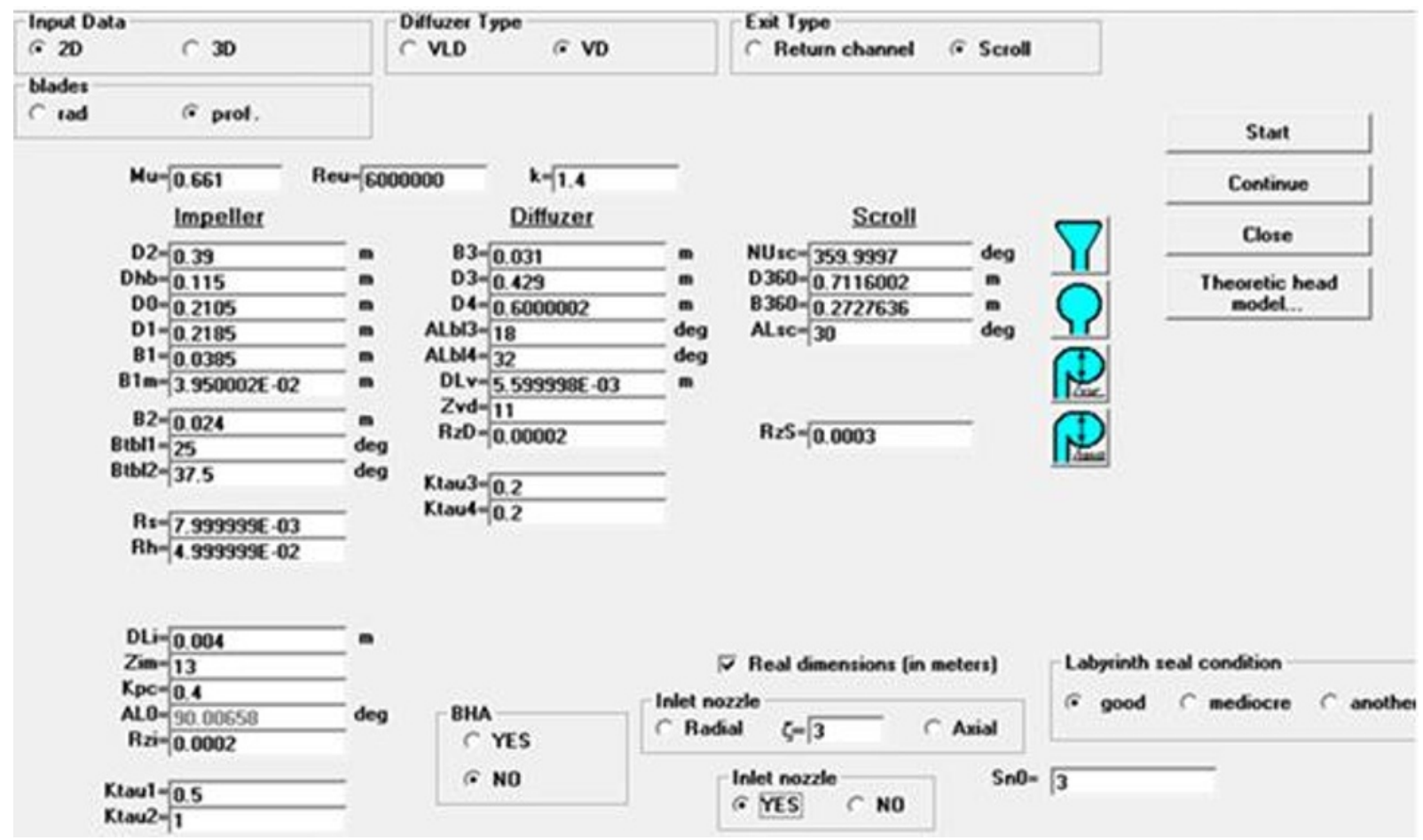

Fig. 1. The CSPM-G8E PC program. Initial parameters and dimensions of the compressor TK-4-530.

\section{8th version of the Universal modeling method math model verification}

The compressor TK-4-530 characteristic calculations were carried out under the following conditions:

- the 8th version standard set of empirical coefficients simulate with good accuracy characteristics of the number of model stages with mechanically treated impellers $[5,8]$. In order to simulate characteristics of the compressor TK-4-530 accurately, the empirical coefficient of the positive incidence losses was increased,

- the surface roughness of the cast impeller was adopted as $0.2 \mathrm{~mm}$, of the cast exit nozzle as $0.3 \mathrm{~mm}$, the mechanically treated vane bladder diffuser as $20 \mu \mathrm{m}$.

Calculations of the characteristics are performed at two Mach numbers of $M_{\mathrm{u}}=0.661$ and 0.858 . It corresponds to points \# 1, $2\left(M_{\mathrm{u}}=0.661\right)$ and \# 13, 14 $\left(M_{\mathrm{u}}=0.858\right)$.

The initial parameters and compressor main dimensions are shown in Figure 1.
Characteristics calculated for two Mach numbers are shown in Figure 2.

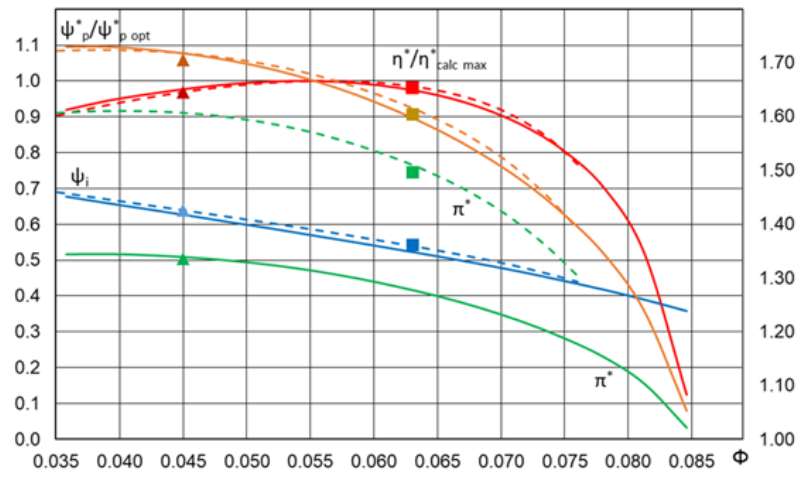

Fig. 2. The compressor TK-4-530 calculated characteristics at $M_{\mathrm{u}}=0.661$ (solid lines), 0.858 (dashed lines) and measured data at $M_{\mathrm{u}}=0.661$ (triangle markers), 0.858 (square markers).

The accuracy of the simulation is demonstrated in Table 4.

The matching of the calculated and measured total

Table 4. Measured parameters of the compressor TK-4-530 at the factory air test.

\begin{tabular}{|c|c|c|c|c|c|}
\hline & $\Phi$ & $\psi_{\mathrm{i}}$ & $\psi_{\mathrm{p}}^{*} \psi_{\mathrm{p} \mathrm{opt}}^{*}$ & $\eta^{*} / \eta^{*}$ calc & $\pi^{*}$ \\
\hline $\begin{array}{c}\text { Measured } \\
/ \\
\text { calculated } \\
\left(\mathbf{M}_{\mathrm{u}}=\mathbf{0 . 6 6 1}\right)\end{array}$ & $\begin{array}{c}0.0450 \\
/ \\
0.0449\end{array}$ & $\begin{array}{c}0.640 \\
/ \\
0.627\end{array}$ & $\begin{array}{c}1.060 \\
/ \\
1.071\end{array}$ & 0.969 & $\begin{array}{c}1.336 \\
/ \\
1.337\end{array}$ \\
\hline $\begin{array}{c}\text { Measured } \\
/ \\
\text { calculated } \\
\left(\mathbf{M}_{\mathbf{u}}=\mathbf{0 . 8 5 8}\right)\end{array}$ & $\begin{array}{c}0.0630 \\
/ \\
0.0626\end{array}$ & $\begin{array}{c}0.543 \\
/ \\
0.542\end{array}$ & $\begin{array}{c}0.908 \\
/ \\
0.923\end{array}$ & 0.981 & $\begin{array}{c}1.496 \\
/ \\
1.502\end{array}$ \\
\hline
\end{tabular}


pressure ratio is almost complete. The matching of other parameters is acceptable for a field-type engineering instrument.

\section{Conclusions}

The specific properties of the compressor TK-4-530 cast impeller are reflected by the new 8th version of the Universal modeling method. It was sufficient to choose properly flow part surfaces' roughness and correct one of dozens empirical coefficients. Following design practice demonstrated that new Universal modeling method is a reliable instrument for design of turboexpander compressors and others process compressors as well.

The calculations were performed using the supercomputer center SCC "Polytechnic". The research was supported by a grant of Russian Science Foundation (project No. 18-7910165).

\section{References}

[1] Z. Galiullin, V. Salnikov, V. Shchurovsky, Modern gas-transportation systems and technologies. Moscow. 345 (2014)

[2] A. Rekstin, V. Semenovskiy, Y. Galerkin, A. Sokolov, The analysis of design and measured gasdynamic characteristics of the centrifugal compressor within turboexpander aggregate - Oil and Gas Engineering (OGE-2018) - AIP Conf. Proc. 2007. Omsk. - Russia. - 2018. 030028-1-030028-11; https://doi.org/10.1063/1.5051889 (2018)

[3] K. Seleznev, Y. Galerkin, E. Popova, Simplified mathematical model of losses in centrifugal compressor stage. Collection of proceedings. Voronezh (1988)

[4] Y. Galerkin, Turbocompressors. LTD information and publishing center KHT. Moscow. 596 (2010)

[5] Y. Galerkin and K. Soldatova, Modeling the working process of industrial centrifugal compressors. Scientific fundamentals, stages of development, current state (Publishing house of Polytechnic University) 327 (2011)

[6] Y. Galerkin and K. Soldatova, The application of the Universal Modeling Method to development of centrifugal compressor model stages. Conference "Compressors and their Systems". London. 477-487 (2013)

[7] Y. Galerkin, A. Rekstin and K. Soldatova Gas dynamic design of the pipe line compressor with 90\% efficiency. Model test approval. Conference "Compressors and their Systems" London (2015)

[8] Y. Galerkin and A. Drozdov, New generation of Universal modeling for centrifugal compressors calculation. Conference "Compressors and their Systems" London (2015)

[9] Y. Galerkin, K. Soldatova, and A. Drozdov, The application of mathematical models for industrial centrifugal compressor optimal design // ICCMS 2018: 10th International Conference on Computer Modeling and Simulation. - Australia. - Sydney. - Paper ID: 008 (2018)

[10] Y. Galerkin, A. Rekstin, K. Soldatova and A. Drozdov, Universal modeling method - the instrument for centrifugal compressor gas dynamic design. ASME Gas Turbine India Conference (2015)

[11] A. Rekstin, Popova Y. and A. Ucehovscy, Centrifugal compressor stages efficiency analysis by means of the approximate algebraic equations. - Oil and Gas Engineering (OGE-2018). - AIP Conf. Proc. 2007. Omsk. - Russia. -. - 030036-1-030036-11 (2018)

[12] Y. Galerkin and K. Soldatova, Universal modeling method application for development of centrifugal compressor model stages. International Conference "Compressors and their Systems". London (2013)

[13] Y. Galerkin, K. Soldatova and A. Drozdov, New version of the universal modelling for centrifugal compressor gas dynamic design. Purdue Conference. USA (2014)

[14] Y. Galerkin, A. Rekstin., K. Soldatova and Drozdov A. Gas dynamic designs of centrifugal compressors for gas industry. Specific features. ASME Gas Turbine India Conference (2015)

[15] Y. Galerkin and A. Drozdov, Centrifugal compressor stage design principlies cheking. ASME Gas Turbine India Conference (2015)

[16] Y. Galerkin, A. Drozdov, A. Rekstin, and Soldatova K., An alternative method for centrifugal compressor loading factor modelling. Conference "Compressors and their Systems" London (2017)

[17] Y. Galerkin, and K. Soldatova, Loading factor performance of a centrifugal compressor impeller. Specific features and way of modeling. World Academy of Science, Engineering and Technology. 18th International Conference on Mathematics and Statistical Engineering. - Australia. - Sydney. 3(12), 822 (2016) 